\title{
VISUAL-IR COLOR GRADIENTS IN ELLIPTICAL GALAXIES
}

\author{
R. F. Peletier, E. A. Valentijn \\ Kapteyn Astronomical Institute, Groningen, the Netherlands \\ R. F. Jameson \\ Department of Astronomy, University of Leicester, United Kingdom
}

Dissipational formation theories (e.g. Larson 1974) predict a metallicity change with distance from the center in elliptical galaxies. Several authors have reported small color gradients in visual colors like $\mathrm{B}-\mathrm{V}$ and $\mathrm{B}-\mathrm{R}$. The interpretation of these data is not easy, due to uncertainties caused by the short wavelength-baseline, and by the presence of a hot stellar population suggested by IUE-data (e.g. Burstein et al. 1986). Simultaneous measurements of visual and visual-infrared colors provide the means to determine both the average temperature of the giant branch and the turnoff-temperature of the main sequence. This allows to model fractional contributions of different populations, including age- and metallicity-effects. The required continuity of solutions at different radii provides a strong constraint in selecting a more unique overall population model, and relieves the ambiguous interpretation of single measurements.

Our observations have been done with the $1 \mathrm{~m}$ Kapteyn Telescope at La Palma with the Leicester Photometer, an instrument measuring a visual and an infrared band simultaneously, making it easier to match the beams. The apertures were pointed at different distances from the center, and their diameters were enlarged at larger radii. We observed NGC 3379,4278 and 5813 in $\mathrm{B}, \mathrm{V}, \mathrm{J}, \mathrm{H}$ and $\mathrm{K}$. All three galaxies show significant color gradients both in visual and visual-infrared colors, in the sense that the galaxies become bluer going outwards. The B-V profiles are consistent with the CCD-photometry by Peletier et al. (1987). Figure 1 shows our data.

Strom et al. (1976) and Tinsley (1978) have made some predictions for V$\mathrm{K}$ gradients as a function of $\mathrm{B}-\mathrm{V}$ gradients in elliptical galaxies. They predict ratios of $\Delta(V-K) / \Delta(B-V)$ of 2.8 (Strom) and 1.6 (Tinsley). Our results of NGC 3379 (2.7) are in agreement with Strom's number. But NGC 4278 (5) and NGC $5813(>10)$ do not agree. They either need an extra hot component in the outer parts or a red component in the center. In the case of NGC 5813 there is good evidence for the presence of dust in the center (Jedrezejewski 1987) and it is well possible that this dust is mainly responsible for the central color gradient. For the three galaxies $\mathbf{M g}_{2}$ line-strength gradients have been obtained by Efstathiou \& Gorgas (1985, NGC 5813) and Davies \& Sadler (1986, NGC 3379 and 4278). We find that our V-J gradients are larger when their $\boldsymbol{M} \boldsymbol{g}_{2}$ gradient is large. A detailed investigation will show how much of the color gradient is due to metallicity differences, to age differences, to dust or to different components which have to be 
included in parts of the galaxy only. A simple empirical approach as employed by Valentijn \& Moorwood (1985) to explain the NGC 3379 data indicates that the main sequence turnoff becomes bluer with increasing radius, while the main giant contribution comes from early $\mathrm{M}$ giants throughout the galaxy.

In the three galaxies we find a correlation between visual-infrared color profiles and the change of position angle and ellipticity. This could give some clues about dynamical mixing, or possibly about merging processes in the past.
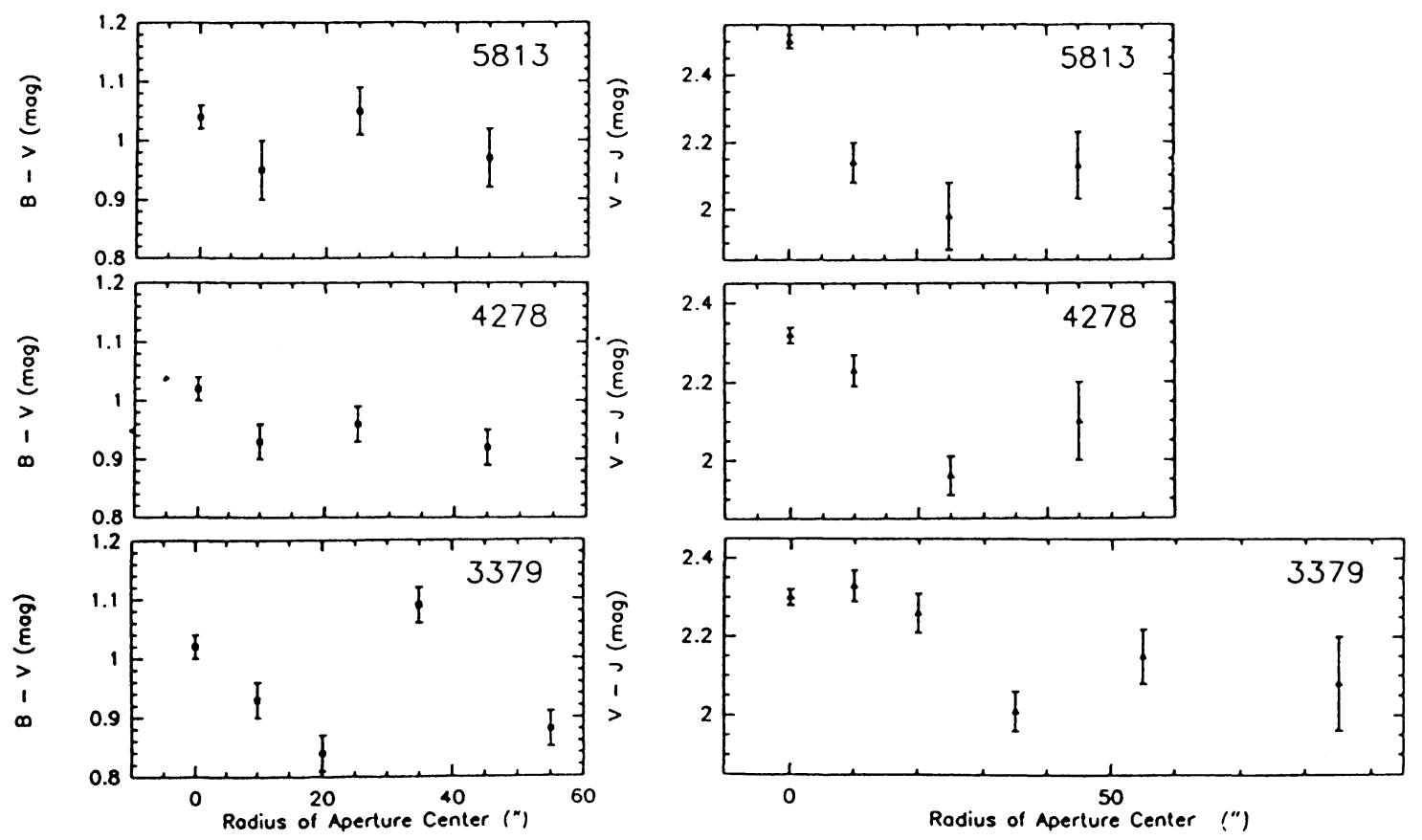

Figure 1. B-V (left) and V-J (right) aperture photometry profiles for NGC 5813, 4278 and 3379.

\section{REFERENCES}

Burstein, D., Bertola, F., Buson, F., Faber, S.M., \& Lauer, T.R., 1986. in Stellar Populations, eds Tosi, M., \& Norman, C., (Baltimore).

Davies, R.L., \& Sadler, E.M., 1986. in Stellar Populations, eds Tosi, M., \& Norman, C., (Baltimore).

Efstathiou, G., \& Gorgas, J., 1985. M.N.R.A.S., 215, 37P.

Jedrezejewski, R., 1987. This volume, p. 37.

Larson, R.B., 1974. M.N.R.A.S., 166, 585.

Peletier, R.F., Davies, R.L., Illingworth, G.D., Davis, L.E., \& Cawson, M., 1987, in preparation.

Strom, S.E., Strom, K.M., Goad, J.W., Vrba, F.J., \& Rice, W., 1976. Astroph. J., 204,684 .

Tinsley, B.M., 1978, Astroph. J., 222, 14.

Valentijn, E.A., \& Moorwood, A.F.M., 1985. Astron. Astroph., 143, 46. 